\title{
Comprehensive precursor anomaly research based on earthquake corresponding relevancy spectrum*
}

\author{
Haitao Wang ${ }^{1,2, \uparrow}$ Qiong Wang ${ }^{2}$ and Lanlan Tang ${ }^{2}$ \\ ${ }^{1}$ Lanzhou Base of Institute of Earthquake Science, China Earthquake Administration, Lanzhou 730000, China \\ ${ }^{2}$ Earthquake Administration of Xinjiang Uygur Autonomous Region, Urumqi 830011, China
}

\begin{abstract}
With the aim to the quantification of anomaly identification and extraction for observed or analyzed records, we present two statistical methods of earthquake corresponding relevancy spectrum (ECRS) and sliding mean relevancy (SMR). With ECRS method, we can obtain the abnormal confidence attribute of data in different value ranges. Based on the relevancy spectrum in different studied time-intervals, we convert the original time sequence into relevancy time sequence, and can obtain the SMR time series by using the multi-point cumulative sliding mean method. Then we can identify the seismic precursor anomaly. We test this method by taking the time sequence of $\eta$-value in the northern Tianshan region as original data. The result shows that when the studied time-interval is 18 months, the precursor anomaly can be identified better from sliding mean relevancy. The anomaly corresponding rate is 83 percent, the earthquake corresponding rate is 86 percent, and the anomaly is characteristic of the middle term. To try the research on multi-parameter comprehensive application, we take the Kalpin tectonic block in Xinjiang as our studied region, and analyze the spatial and temporal abnormal characters of multi-parameter sliding extreme-value relevancy (MSER) before mid-strong earthquakes in the Kalpin block. The result indicates that ECRS and SMR sequence in different time-intervals can not only be used to identify the precursor anomaly of single-item data, but also offer the data of quantitative single-item anomaly for comprehensive earthquake analysis and prediction.
\end{abstract}

Key words: earthquake corresponding relevancy spectrum(ECRS); sliding mean relevancy (SMR); multi-parameter sliding extreme-value relevancy(MSER); comprehensive precursor anomaly

CLC number: P315.72 Document code: A

\section{Introduction}

Precursory anomaly identification of observed or analyzed records is the key for earthquake prediction research and practice. Large numbers of earthquake cases indicate that the observed records of different types such as strain, geoelectricity, geomagnetism, and groundwater etc., and many seismic parameters deriving from earthquake catalogues such as $b$ value, $c$ value, $D$ value, $\eta$ value etc., may appear as precursory anomalies of different shapes before the big earthquakes to some extent. Researchers usually judge whether the observed and analyzed records for some period are anomalous according to the character of different shapes. For ex-

\footnotetext{
* Received 16 June 2009; accepted in revised form 10 September 2009; published 10 December 2009.

- Corresponding author. e-mail: ht.wang @263.net
}

ample, "rising-high value variation-dropping-occurring" and "dropping-low value variation-rising-occurring", which are two types of common precursory anomaly, appear widely in many articles. Even to the different events of the same time curve, the lasting time and magnitude of anomaly variation is also different. It is commendable to conclude and extract the anomalous character based on experienced and qualitative analysis prediction. However, the multi-method comprehensive analysis should be done based on quantitative description of single-subject anomaly. Therefore, researchers try to employ several methods to quantitatively identify and extract observed and analyzed records, and convert an original curve into a new curve with the some standardization-meaning. The new curves not only intensify precursory anomaly process with new patterns, but also provide the quantitative basal data for different com- 
prehensive analysis and prediction models. For example, Wang et al (1991) employed the distribution probability method to preprocess radon content in underwater curves, and calculated the comprehensive comentropy. Bo and Wang (1993), Bo and Wu (1993) proposed the multi-point slope and synthetic information flow method, and converted strain records into "standardization information curves". Zheng and Feng (1993) converted precursory records into anomaly dynamic membership functions using the vague mathematics method. Du et al (2001) normalized resistivity records with the monthly rate method, and got new non-dimensional data. There are lots of similar methods. With relative research illumination, the author presents statistical methods which takes earthquake corresponding relevancy spectrum (ECRS) based on statistical analysis of single-item observed and analyzed data time sequence. This tries to effectively identify precursory anomalies, and provides one new single-factor quantitative method for application research on earthquake comprehensive prediction methods.

Based on the single parameter research, the article selects the different seismic parameters, such as: the $\eta$ value standing for the deviation between magnitude-frequency curve and $G-R$ relation (Utsu, 1980), $b$ value for earthquake energy distribution, $D$ value for seismic risk factor (Wang, 1987), $\bar{M}$ for mean magnitude (earthquake deficiency, Wei, 1980), $M_{f}$ value for seismic intensity factor (Wang et al, 1994), $N$ for frequency and $R_{\mathrm{m}}$ value for regulatory ratio of small earthquake (Qin et al, 1983), which represent the different characters of seismic activity, to calculate multi-parameter sliding extreme-value relevancy (MSER), and primarily identify the spatial and temporal characters of comprehensive precursor anomalies before mid-strong earthquakes. This provides one new quantitative method for comprehensive prediction research.

\section{Method}

\subsection{Value range spectrum analysis}

For the observed record sequence $x_{1}, x_{2}, \ldots, x_{n}$, the maximum value of the sequence is $x_{\max }$, and the minimum value is $x_{\min }$. The mean value and standard deviation of the sequence is as follows

$$
\bar{x}=\frac{1}{n} \sum_{i=1}^{n} x_{i}
$$

$$
\sigma=\sqrt{\frac{\sum_{i=1}^{n}\left(x_{i}-\bar{x}\right)^{2}}{(n-1)}}
$$

Based on the mean value and standard deviation, the rule of value range distribution interval is defined as follows

$$
\begin{gathered}
x_{0} \in(\bar{x}-k \sigma, \bar{x}+k \sigma), \\
x_{1} \in[\bar{x}+k \sigma, \bar{x}+2 k \sigma), \\
x_{2} \in[\bar{x}+2 k \sigma, \bar{x}+3 k \sigma), \\
x_{m} \in\left[\bar{x}+m k \sigma, x_{\max }\right], \\
x_{-1} \in(\bar{x}-2 k \sigma, \bar{x}-k \sigma], \\
x_{-2} \in(\bar{x}-3 k \sigma, \bar{x}-2 k \sigma], \\
x_{-m} \in\left[x_{\min }, \bar{x}-m k \sigma\right] .
\end{gathered}
$$

According to formulae (3) through (9), the frequency of the observed value sequence $x_{i}$, which distributes in the corresponding interval, is counted. Then the value range spectrum curve is obtained, which is applied to the analyzed data structure, and revises the parameters $k$ and $n$. If the sequence is stable, then the $k$ value may be smaller, such as 0.15 or so. If the sequence fluctuates, then $k$ value may be bigger, such as 0.3 or so. The $m$ value is usually assigned to a number between 6 and 10. During the data processing, the $k$ and $m$ values could be revised accordingly since the value range spectrum curve is similar to the normal distribution. When the interval distribution of some data is asymmetrical when compared to mean value, then formulae (3) to (6) and formulae (7) to (9) may be assigned different $k$ values, so as to obtain a value range spectrum similar to the normal distribution.

\subsection{Earthquake corresponding relevancy spectrum analysis (ECRS)}

Based on the above work, we can calculate the ECRS of different value ranges with different studied time-intervals. First, we select the earthquake magnitude threshold for the target earthquake of our testing prediction, which is related to the analysis data in some spatial range, i.e., the earthquakes greater than 5.0 are taken as target events of retrospective prediction tests. Secondly, we count, point by point, the observed data frequency distributing in the different interval of $x_{-\mathrm{m}}$ to $x_{\mathrm{m}}$, and the target earthquakes in the different studied time-interval. According to the above rule, we count observed data points $n_{\mathrm{m}}$ which correspond target earthquakes and data 
points in the matching value range $N_{\mathrm{m}}$, and calculate the ratio (called as earthquake corresponding relevancy). By counting all the value ranges, we obtain the ECRS within the different value ranges of observed data sequences, i.e., $r_{\mathrm{m}}=n_{\mathrm{m}} / N_{\mathrm{m}}$. We can not only apply the earthquake corresponding relevancy to analyze basic anomaly characters of original observed data, or judge anomaly attributes in different value ranges, but also the earthquake corresponding relevancy provides basic data for further calculation.

\subsection{Data conversion and sliding mean relevancy (SMR)}

Supposing that $t$ is some different time-interval (unit: month); and $j$ is some different seismic parameter.

Based on ECRS, we can convert the observed original time sequence into relevancy time sequence $r_{i j}$ in different studied time-intervals according to the point-to-point belonged value range. Then we can obtain the SMR time sequence $\bar{R}_{i j}$ by using the multi-point cumulative mean and the point-to-point sliding method. By analyzing corresponding SMR, we may identify seismic precursor anomalies.

$$
\begin{gathered}
\bar{R}_{i j}=\frac{\left(r_{i j}+r_{(i+1) j}+\cdots+r_{(i+t-1) j}\right)}{t} \\
i=1,2, \cdots, n ; t=3,6,12,18 ; j=1,2, \cdots,
\end{gathered}
$$

\subsection{Multi-parameter sliding extreme-value rele-} vancy analysis (MSER)

There are different abnormal shapes with different seismic parameters presented before a mid-strong earthquake, i.e., anomaly starting times, peak values, and ending times are different. To strengthen mid-short term precursor abnormal process before mid-strong earthquake, the article presents the multi-parameter sliding extreme-value relevancy (MSER) based on identifying single-parameter anomalies.

By calculating multi-point extreme values of SMR with different parameter $M_{i j}$, we obtain the sliding ex- treme-value relevancy sequence $M_{j}$ of every parameter. Then we can calculate the mean of the parameter $M_{j}$, and have the MSER value $R_{\mathrm{c}}$.

Suppose that $w$ is the point number calculating the extreme value. $w$ is assigned to 6 in our study.

$$
\begin{gathered}
M_{i j}=\max \left\{\bar{R}_{i j}, \bar{R}_{(w+1) j}, \cdots, \bar{R}_{(i+w-1) j}\right\} \\
i=1,2, \cdots,(n-w+1), \\
M_{j}=\left(M_{1 j}, M_{2 j}, \cdots, M_{(n-w+1) j}\right) \\
j=1,2, \cdots k, \\
R_{\mathrm{C}}=\frac{\sum_{i=1}^{k} M_{j}}{k} .
\end{gathered}
$$

\section{Case analysis}

To test this method we must first research and analyze the seismic parameter $\eta$-value time sequence in the northern Tianshan region. Secondly, we must analyze the temporal abnormal character of MSER before mid-strong earthquakes in the Kalpin block. Lastly, we must analyze the spatial abnormal character of MSER before and after the 2005 Wushi earthquake of $M_{\mathrm{S}} 6.3$ in the Kalpin block. The article selects the $M_{\mathrm{S}} \geq 5.0$ earthquakes in studied regions as prediction target events.

\subsection{SMR case analysis}

Figure 1 is the time curve for the $\eta$ value in the northern Tianshan region with twelve-month intervals and one-month sliding step. There were eight $M_{\mathrm{S}} \geq 5.0$ earthquakes that occurred in the studied time-interval. The short-term anomaly character is low-value variation, and medium and long term character is "high value-dropping (rising)-earthquake occurring" variation before the target earthquake. Also, no earthquakes occurred after the similar characters appear.

According to the above method, we show the calculating process step by step as follows. Figure 2 is the corresponding value range spectrum curve based on the

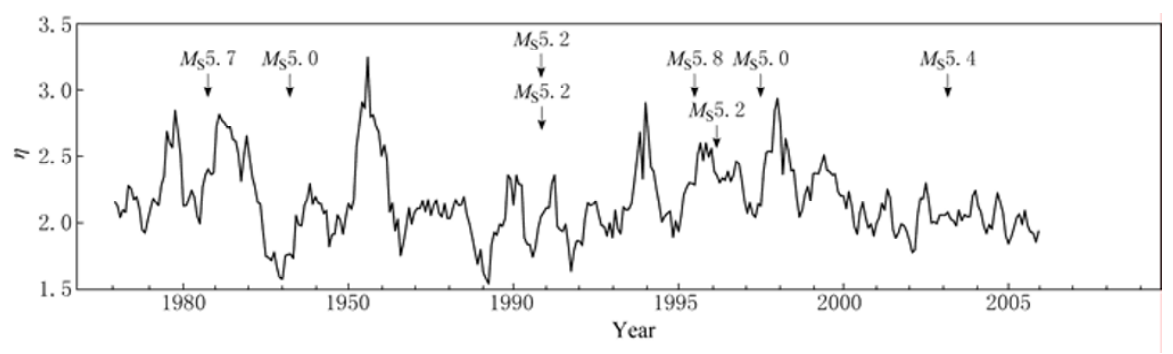

Figure 1 Time curve for the $\eta$-value in the northern Tianshan region. 
time sequence for the $\eta$ value in the northern Tianshan region. The horizontal axis is value range, and 0 represents the value range of $x_{0}$ (i.e., mean value range), 6 represents the value range of $x_{6},-6$ represents the value range of $x_{-6}$, and so on. The vertical axis is the data frequency distributing in the corresponding value range. Figure 2 shows that the value range spectrum is similar to normal distribution, which indicates that the value range distribution is reasonable, i.e., $n=9, k=0.25$.

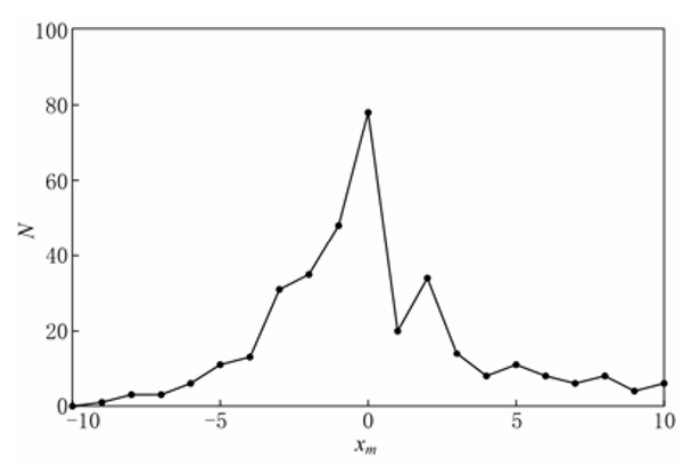

Figure 2 Value range spectrum curve.

To analyze the precursor variation character of different anomaly efficiency of SMR of the $\eta$ value, we select the fitful time-interval. This identifies precursor anomaly efficiently by analyzing the different studied time-intervals, e.g., three months, six months, 12 months, 18 months and 24 months. The article discusses the result of the studied time-intervals of six months and 18 months.

Figure 3 is the curve of ECRS of the $\eta$ value with the six-month time-interval and six-point sliding mean relevancy. Figure $3 \mathrm{a}$ is the ECRS of the $\eta$ value. The horizontal axis is the same as Figure 2, and vertical axis is earthquake corresponding relevancy of different value ranges with the six-month time-interval (i.e., the ratio of the count of corresponding earthquake to the count in the same value range). Figure $3 \mathrm{~b}$ is the point-point SMR curve of the $\eta$ value with six-point step, based on point-point value range of the Figure 1 curve and relevancy converting according to ECRS of Figure $3 \mathrm{a}$.

Figure $3 \mathrm{a}$ indicates that the precursor anomaly confidence of the $\eta$ value near to the mean value is lower, and the confidence of $\eta$ value in the low value range is higher. This means that the $\eta$ value in the studied region is characteristic of low value six months before the target events according to ECRS, which is in accord with the fact.

Taking the values obviously greater than the mean value as anomaly criteria (shadow area in the Figure $3 \mathrm{~b}$ ), there are seven sets of anomaly variation. Also, earthquakes occurred during three of the seven sets of high value variation, and the anomaly corresponding ratio was 43 percent. The eight target earthquakes can be taken as seven sets (1990 earthquakes can be taken as one set). There were anomaly variations before three of the seven sets of earthquakes, and the earthquake corresponding ratio was 43 per cent. According to the SMR with six-month time-interval, we may identify precursor anomaly characters, but the confidence is not high, and the false and missing prediction ratio may be high with such anomaly criteria.
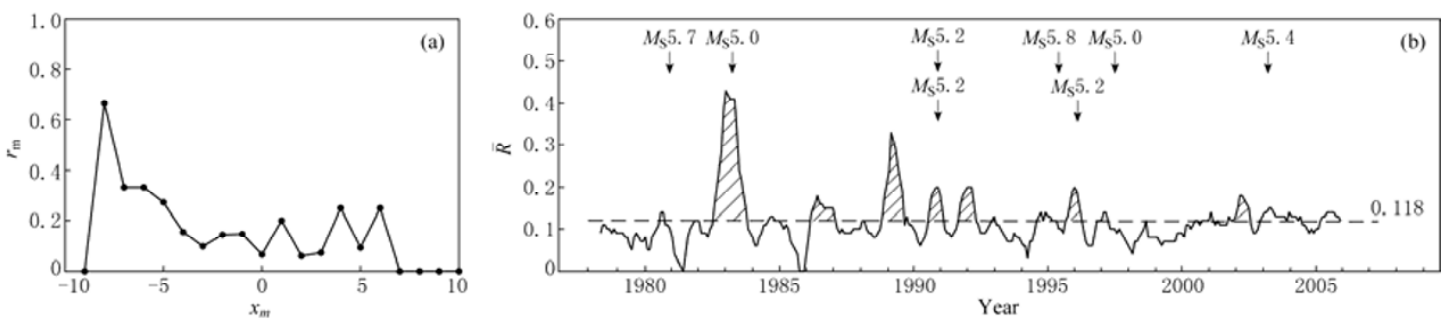

Figure 3 The ECRS and SMR curve of the $\eta$ value with six-month time-interval in the northern Tianshan region. (a) ECRS; (b) SMR time curve.

Figure 4 is the ECRS of the $\eta$ value and 18-point SMR curve with 18-month time-interval.

Figure $4 \mathrm{a}$ indicates that the anomaly confidence of the $\eta$ value in the low and high value range is high. The confidence of the high value fluctuates, but the confidence of the low value is high and stable.

Taking the values obviously greater than the mean value as anomaly criteria (shadow area in the Figure $4 b$ ), there are six sets of anomaly variation. Also, earthquakes occurred during five of the six sets of high value variation, and the anomaly corresponding ratio was 83 percent. There were anomaly variations before six of the seven sets of target earthquakes, and the earthquake corresponding ratio was 86 percent. According to the 
SMR with 18-month time-interval, we can not only identify precursor anomaly characters, but also the con- fidence of anomaly and earthquake corresponding ratio was high with such anomaly criteria.
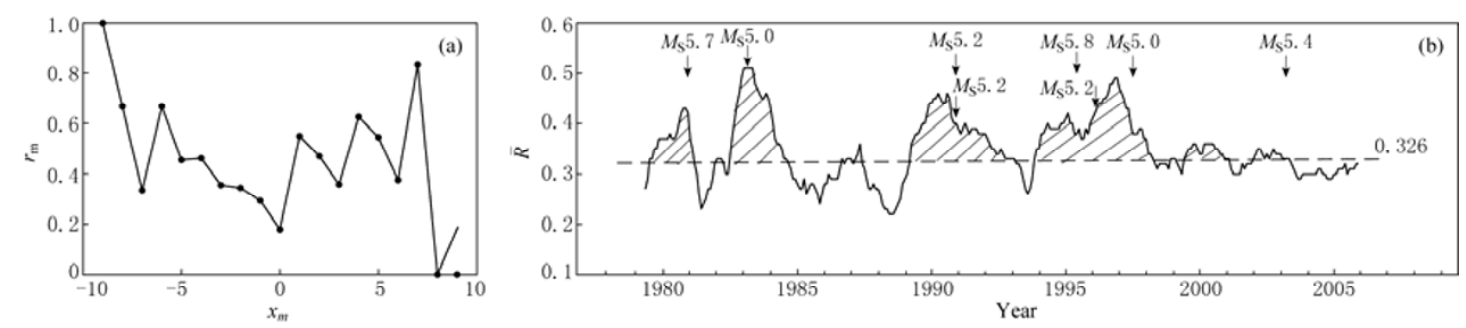

Figure 4 The ECRS and SMR curve of the $\eta$ value with 18-month time-interval in the northern Tianshan region. (a) ECRS, (b) SMR time curve.

\subsection{MSER analysis}

Based on single-parameter analysis, the article selects different seismic parameters of $\eta$ value, $b$ value, $D$ value, earthquake deficiency, $M_{f}$ value, frequency and regulatory ratio of small earthquakes $R_{\mathrm{m}}$. It then analyzes the spatial and temporal character of comprehensive anomaly of MSER with 18-month intervals in the Kalpin block, Xinjiang.

1) Case analysis on temporal character. Figure 5 is the curve for the MSER with an 18-month interval in the
Kalpin block. The article calculates the extreme value of SMR for different parameters with the step of six-point, and then gets the MSER value $P_{\mathrm{C}}$. There are four abnormal processes in the studied time in the Kalpin block, and there are target earthquakes occurrences during the four high relevancy processes. There exist obvious abnormal processes of high relevancy before four processes of the six target earthquakes with $M_{\mathrm{S}} \geq 5.5$ in the studied time.

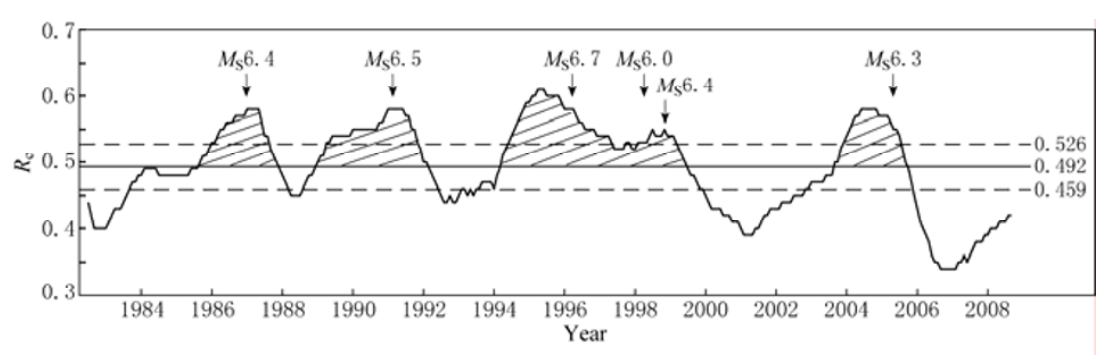

Figure 5 The MSER with 18-month interval in the Kalpin block.

2) Case analysis on spatial character. The article calculates the monthly spatial distribution data of MSER before and after the 2005 Wushi earthquake of $M_{\mathrm{S}} 6.3$ in the Kalpin block, Xinjiang, with the 18-month intervals, by scanning in the $2^{\circ} \times 2^{\circ}$ cell size and every one step of $0.2^{\circ}$.

The detailed steps are as follows, firstly, we converted the monthly spatial distribution data of different seismic parameters with an 18-month interval into monthly spatial value of ECRS and SMR. Secondly, based on monthly spatial values of SMR of different parameters, we calculated monthly spatial values of the multi-point sliding extreme-value relevancy of every parameter. Lastly, by calculating the mean of the multi-point sliding extreme-value relevancy of different parameters, we obtained spatial distribution data of the multi-parameter sliding extreme-value relevancy (MSER).

Figure 6 shows maps for MSER before and after the 2005 Wushi earthquake of $M_{\mathrm{S}} 6.3$. The MSER appears small high-value abnormal area since January, 2004. Then the abnormal area started to centralize and extend in the seismical region of the Wushi $M_{\mathrm{S}} 6.3$ earthquake. The high relevancy in the seismical region started to weaken after the strong aftershock of $M_{\mathrm{S}} 5.0$, and the abnormal area shrunk and disappeared one year after the Wushi $M_{\mathrm{S}} 6.3$ earthquake. The high relevancy abnormal area in the lower part of Figure 6 may be related to the Uzbel Mountain pass $M_{\mathrm{S}} 5.0$ earthquake of March 21, 2004, and the Tajikistan $M_{\mathrm{S}} 5.5$ earthquake of February, 2005 in the surrounding region. 
The spatial abnormal character of MSER in the Kalpin block showed that high-value anomaly confidence is high, i.e., target earthquakes occurred during the high-value process. However there is missing prediction; there were no high-value anomalous process before the two processes of the six target earthquakes $M_{\mathrm{S}} \geq 5.5$.
The spatial abnormal character showed that the MSER value started to appear high-value anomalous area one year or so before the 2005 Wushi earthquake of $M_{\mathrm{S}} 6.3$, and tended to extend and intensify to the seismical region. Also, the high-value area started to weaken and disappeared one year or so after the Wushi $M_{\mathrm{S}} 6.3$ earthquake.
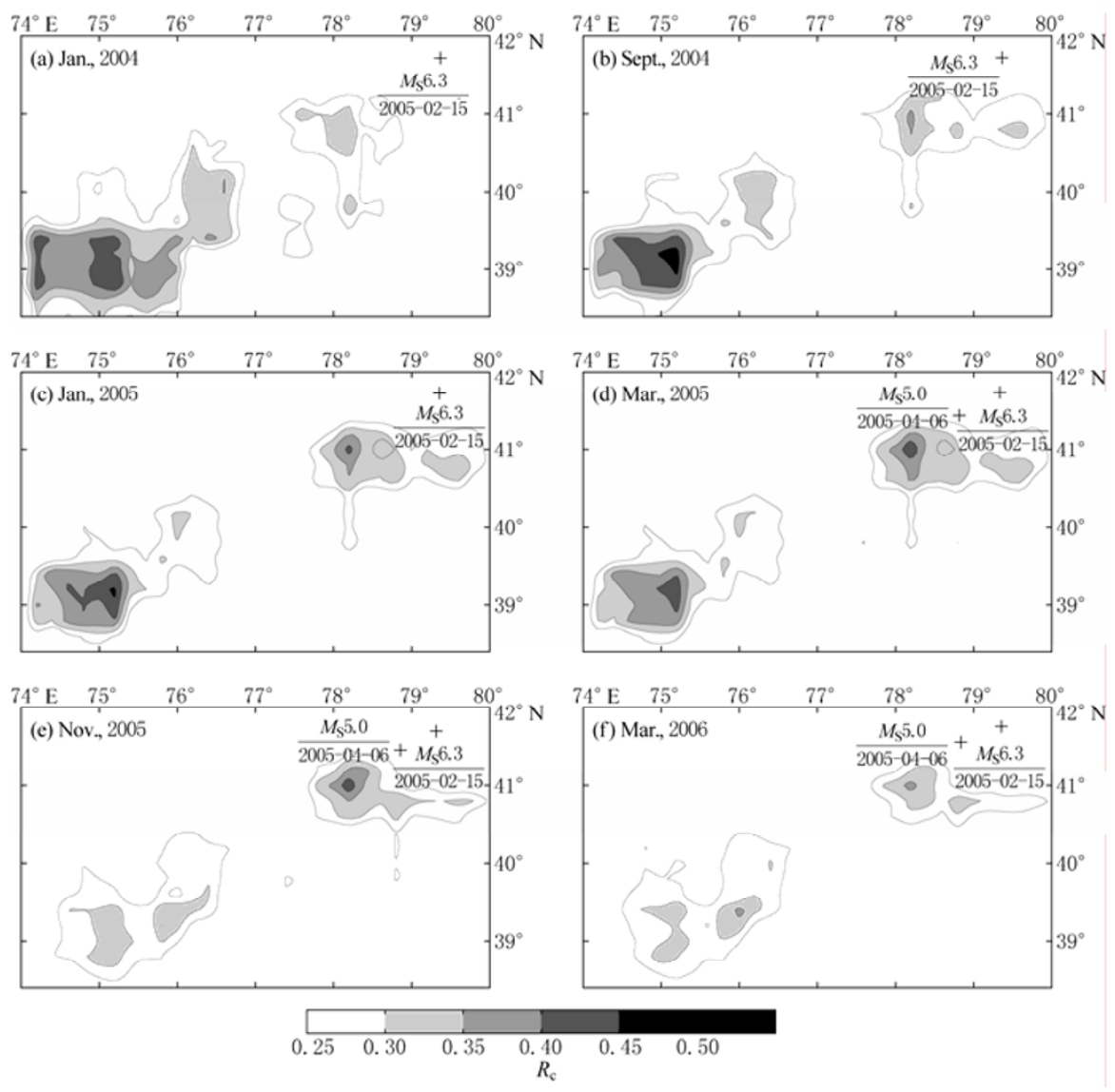

Figure 6 Maps for MSER before and after the 2005 Wushi earthquake of $M_{\mathrm{S}} 6.3$.

\section{Discussion and conclusions}

The article applies the seismic parameter time sequence in northern Tianshan region to calculate and analyze earthquake corresponding relevancy spectrum (ECRS) in different studied time-intervals. The result shows that precursor anomaly efficiency attributes of ECRS with different studied time-intervals are different. SMR analysis based on the earthquake corresponding relevancy(ECRS) indicates that SMR with the 18-month time-interval in the northern Tianshan region has higher anomaly corresponding ratio and earthquake corresponding ratio than the 6-month one, which shows that the method can identify middle -term precursor anoma- lies in the studied region.

The temporal abnormal character of MSER with an 18-month interval in the Kalpin block shows that high-value anomaly confidence is high, i.e., target earthquakes occurred during the high-value process. However, there is missing prediction; there were no high-value anomalous processes before the two processes of the six target earthquakes with $M_{\mathrm{S}} \geq 5.5$. Therefore, how to improve anomaly extraction efficiency will be the further work of our future study.

The spatial abnormal character showed that the MSER value started to appear high-value anomalous area one year or so before the 2005 Wushi earthquake of $M_{\mathrm{S}} 6.3$, and tended to extend and intensify to the seismi- 
cal region, and the high-value area started to weaken and disappeared one year or so after the Wushi $M_{\mathrm{S}} 6.3$ earthquake.

The ECRS is the key problem in our study. Taking the different time-interval corresponding relevancy from the retrospective test prediction analysis of history data as comparing database, the database may provide quantitative single-factor data of different prediction times for comprehensive analysis and prediction.

Comprehensive analysis of seismic precursor anomalies is necessary for earthquake prediction research and practice. The key of comprehensive analysis and prediction is to efficiently identify and extract anomalous criterion of the single-item prediction method. The article employs the new methods of ECRS and SMR to analyze the practical data. The primary result shows that these methods can be applied to analyze the precursor anomaly efficiency attribute of observed or analyzed records. This is significant to improve the time-predicted efficiency on comprehensive analysis and prediction.

These methods can be applied directly to identify and research precursor anomalies of the stable observed and analyzed time sequence. The data, which has trend variation, periodical annual variation, or distortion point, should be preprocessed by eliminating trend, fitting residual error, and removing distortion point by interpolation etc.. It is necessary for these methods to have some target earthquakes as corresponding analysis sample. So it is, to some extent, limited. To temporal character analysis, we can enlarge the target earthquake number by selecting studied areas. To spatial character research, if some spatial areas have no target earthquakes, then how to solve the problem is something to give further thought.

Based on retrospective case research, we can obtain a good retrospective prediction result by revising calculating parameters. However, because using the latter data as comparing criteria of former data, the result is, to some extent, unreasonable. This is one of the reasons why retrospective prediction efficiency is worse than the real situation. It is also unavoidable to do the related research with few samples of big earthquakes and studied data which is short-duration and limited.

With the accumulation of modern seismic observational data, the related research may use the long history data as comparing data for retrospective analysis, and may use earthquake correspondence and anomaly correspondence in the retrospective studied phase as retrospective test criteria. The extrapolation prediction test should be calculated within some time phase, which can avoid using the data after target earthquake to contribute reversely calculation and statistic analysis before the target earthquake (e.g., mean calculation). Then the extrapolation prediction result will be more significant to guide earthquake prediction practices.

The article introduces the two statistical methods of earthquake corresponding relevancy spectrum (ECRS) and sliding mean relevancy (SMR) with seismic parameters, and aims to exploring one new comprehensive method identifying and extracting the anomaly information. The result is the primary research of one subject of a national key scientific and technological project we have undertaken, and it is necessary to study further, especially selecting rational parameters, with the calculation and analysis of more data. To dynamic evolvement character on multi-parameter comprehensive anomaly before mid-strong earthquakes, we plan to research further in future.

Acknowledgements We appreciate the constructive reviews of anonymous reviewers on the manuscript. We thank Dr Xiaodong Song for his assistance with English writing of the manuscript and helpful comments.

\section{References}

Bo W J and Wang Y (1993). One new method to extract anomalous variation of single-item data. Journal of Geodesy and Geodynamics 9(3): 17-20 (in Chinese with English abstract).

Bo W J and Wu Y L (1993). Standard method and application of anomalous information flow. Deformation and Earthquake 13(2): 9-15 (in Chinese with English abstract).

Du X B, Ruan A G and Fan S H (2001). Anisotropy of resistance variation rate near the epicenter region of strong earthquake. Acta Seismologica Sinica 23(3): 289-297 (in Chinese with English abstract).

Qin B Y, Xu J R, Yao L X and Li Y R (1983). Regulatory feature of small earthquake in Xihaigu region. Northwestern Seismological Journal 5(Suppl.): 1-5 (in Chinese with English abstract).

Utsu T (1980). Parameter estimation in earthquake frequency and magnitude expression-including maximum magnitude parameter $c$. Translated World Seismology 3: 23-31.

Wang H T, Zhu L R and Yang M L (1991). Analysis on information entropy of seismic precursor observed data. Earthquake 11(5): 13-18 (in Chinese with English abstract).

Wang W (1987). The abnormal variation of seismic risk factor $D$ vaule before 5 mid-strong earthquakes in North China. Earthquake 7(1): 17-25 (in Chinese with English abstract).

Wang W, Dai W L and Huang S B (1994). Statistical distribution of earthquake magnitude and mid-term abnormal variation of $M_{\mathrm{f}}$ value for seismic intensity factor before mid-strong earthquakes in North China. Earthquake Research in China 10(Suppl.): 95-110 (in Chinese with English abstract).

Wei P F (1980). Earthquake deficiency curve in North China region. Journal of Seismological Research 3(4): 144-148 (in Chinese with English abstract).

Zheng X M and Feng D Y (1993). Spatial and temporal evolution of precursor anomaly and seismic activity. Deformation and Earthquake 13(2): 80-87 (in Chinese with English abstract). 